\title{
On The Electric Fields And Currents Produced By Induction Logging Instruments In Anisotropic Media
}

Xinyou Lu', David L. Alumbaugh' and ChesterJ. Weiss ${ }^{2}$

'Geological Engineering Program, University of Wisconsin-Madison, 1415 Engineering Drive, Madison, WI 53706.

${ }^{2}$ Geophysical Technology Department, Sandia National Laboratories, PO Box 5800 MS-0750, Albuquerque NM 87185.

\begin{abstract}
In this paper, we investigate the electric fields and currents induced by a time-harmonic horizontal magnetic dipole source embedded in a transversely anisotropic conducting medium. The medium is uniform wholespace characterized by a "horizontal conductivity" which is constant in each of the two horizontal directions and "vertical conductivity" for the third, vertical, direction. The results of this investigation are assessed using two- and three-dimensional visualization techniques. Renderings of these vector fields and their associated streamlines visually demonstrate the well-known pattern of coaxially aligned circular current paths in the isotropic limit where the vertical and horizontal conductivties are equal. Our investigation also demonstrates that moderate values $(2$ to $\sim 5)$ of the ratio of horizontal to vertical conductivity generate induced electric currents which are no longer coaxially aligned, acquiring a component in the direction of the dipole moment of the source. For ratio values in excess of $\sim 10$, our results indicate that the horizontal components of the electric and current density fields dominate the vertical components, giving rise to closed horizontal loops of electric current. An explanation of the underlying physics of this phenomenon is then offered, based on analogy with the induced electric fields within a conducting isotropic halfspace.
\end{abstract}

\section{INTRODUCTION}

For several decades now electrical anisotropy has attracted the interest of the induction logging community in their efforts to better characterize potential hydrocarbon reservoirs (e.g. Kunz and Moran, 1958; Moran and Gianzero, 1979; Anderson et al., 1998). In terms of the interpretation challenge faced by the log analyst, electrical anisotropy arises from the presence of sedimentary structures whose length scale is far smaller than the resolving power of the induction sonde. For example, shales and thinly laminated sequences of shale and sand are commonly-encountered anisotropic formations (Klein et al., 


\section{DISCLAIMER}

This report was prepared as an account of work sponsored by an agency of the United States Government. Neither the United States Government nor any agency thereof, nor any of their employees, make any warranty, express or implied, or assumes any legal liability or responsibility for the accuracy, completeness, or usefulness of any information, apparatus, product, or process disciosed, or represents that its use would not infringe privately owned rights. Reference herein to any specific commercial product, process, or service by trade name, trademark, manufacturer, or otherwise does not necessarily constitute or imply its endorsement, recommendation, or favoring by the United States Government or any agency thereof. The views and opinions of authors expressed herein do not necessarily state or reflect those of the United States Government or any agency thereof. 


\section{DISCLAIMER}

Portions of this document may be illegible in electronic image products. Images are produced from the best available original document. 
1995). In addition, variation in the grain size distribution within layered sandstones can generate electrical anisotropy since the macroporous layers desaturate more quickly than the microporous layers, and thus become more resistive (Klein et al., 1995). These types of formations are likely to exhibit one value of conductivity parallel to the bedding planes, and a different, lesser, value of conductivity perpendicular to beds. This is the case of transverse isotropy. For typical reservoir rocks, Klein et al. (1995) show that the ratio of horizontal to vertical conductivity can be as large as 100 . Fortunately enough, the effects of anisotropy can safely be ignored in situations where the orientation of the borehole axis is perpendicular to any fine-scaled sedimentary bedforms.

Historically, exploration wells were drilled vertically and were often perpendicular to formation bedding planes. In this situation, conventional induction tools induce eddy currents that flow horizontally, i.e. parallel to the bedding planes. Even though the horizontal and vertical conductivity may differ, coaxial induction instruments are only sensitive to the horizontal formation conductivity. This is the primary reason why electrical anisotropy has been largely ignored by many log analysts. However, deviated and horizontal wells have been increasingly utilized in the last $\sim 10$ years to better characterize the reservoir and lower exploitation costs. As a result of this new drilling strategy, the eddy currents induced in the formation by a sonde located in a deviated or horizontal well are forced to cross bed boundaries and, thus, are sensitive to both the vertical and horizontal conductivity of the formation. Increased attention has been devoted to the anisotropy problem as evidenced by recent efforts to initiate large scale discussion and collaboration through a workshop on borehole and formation anisotropy, organized by the Society of Professional Well Log Analysts in Corpus Christi, Texas, on April 2-6, 2000. Given this simple conceptual framework, analysis of the anisotropic effects due to a horizontal magnetic dipole is essential to understanding the response of the induction sonde in a dipping well.

The problem of induction of a horizontal magnetic dipole source in the presence of anisotropic whole space or layered anisotropic media has been solved analytically by Sinha (1968), Kong (1972, 1975), Tang (1979), Moran and Gianzero (1979) and others. However, even though the solutions have been available for quite some time, a discussion of how anisotropy distorts the electric fields and currents has not been presented as far as we know. The electric field and current patterns are complicated and not well understood. Thus, in this paper, we study the effects of anisotropy on the electric fields and currents produced by a horizontal magnetic dipole. In Section 2 we provide the anisotropic model on which this

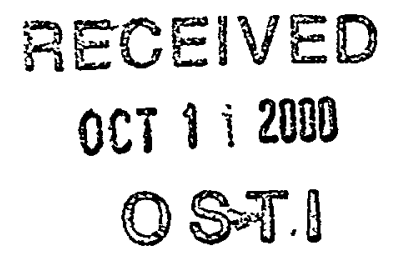


study is based, and outline an analytic solution for this model. In Section 3 we visualize the electrical fields and currents induced by a horizontal magnetic dipole within anisotropic media to demonstrate the effects that the anisotropy can have on the fields and currents. Here both two-dimensional (2D) and three-dimensional (3D) visualization is employed to graphically display these electromagnetic phenomena.

\section{MODEL}

The model considered in this paper is that of transverse isotropy as shown in Figure 1. The resistivity in the two horizontal directions ( $x$ and $y$ ) is constant, but varies in the vertical direction. The anisotropy can be described by the coefficient of anisotropy $(\lambda)$, which is the square root of the ratio of the vertical resistivity $\left(R_{v}\right)$ to horizontal resistivity $\left(R_{h}\right)$ and is defined as $\lambda^{2}=R_{v} / R_{h}=\sigma_{h} / \sigma_{v}$, where $\sigma_{v}$ and $\sigma_{h}$ are vertical and horizontal conductivities. The magnetic permeability $(\mu)$ and dielectric permitivity $(\varepsilon)$ are assumed to be isotropic and set to their free-space values such that any perturbation in the fields will be due only to anisotropy within the formation. This transversely isotropic case is simple, but very practical as sedimentary rocks at depth tend to exhibit this type of electrical structure. Analytic solutions for this model are readily available if the effects of the borehole and invasion zone can be ignored. Moran and Gianzero (1979) showed that for a transversely isotropic whole space, the model can be represented by a tensor of the following form:

$$
\overline{\bar{\sigma}}=\left[\begin{array}{lll}
\sigma_{\mathrm{h}} & & \\
& \sigma_{\mathrm{h}} & \\
& & \sigma_{\mathrm{v}}
\end{array}\right] .
$$

Readers are referred to Moran and Gianzero (1979) for details on derivation, which employs a Cartesian coordinate system with its origin at transmitter location and $z$ positive upwards. For this system, the Hertz potentials in the frequency domain for an $x$-directed horizontal magnetic dipole with the moment of $M$ are: 


$$
\bar{\pi}=\left[\begin{array}{c}
\frac{M}{4 \pi \lambda} \frac{e^{i k_{v} s}}{s} \\
0 \\
\frac{M}{4 \pi} \frac{x}{\rho^{2}}\left(\lambda z \frac{e^{i k_{v} s}}{s}-z \frac{e^{i k_{h} r}}{r}\right)
\end{array}\right],
$$

and

$$
\Phi=\frac{M}{4 \pi} \frac{i k_{h} x}{\rho^{2}}\left[e^{i k_{v} s}-e^{i k_{h} r}+\frac{\rho^{2}}{r^{2}}\left(1-\frac{1}{i k_{h} r}\right) e^{i k_{h} r}\right]
$$

where

$$
\begin{aligned}
& k_{h}^{2}=i \omega \mu \sigma_{h}, \\
& k_{v}^{2}=i \omega \mu \sigma_{v}, \\
& r=\sqrt{x^{2}+y^{2}+z^{2}}, \\
& s=\sqrt{x^{2}+y^{2}+\lambda^{2} z^{2}}, \\
& \rho=\sqrt{x^{2}+y^{2}} .
\end{aligned}
$$

Note here a time dependence of $e^{-i \omega t}$ is assumed. Then electric fields can be calculated by

$$
\overline{\bar{\sigma}} \bar{E}=i \omega \mu \sigma_{h} \nabla \times \bar{\pi}
$$

or 


$$
\begin{aligned}
E_{x}= & \frac{i \omega \mu M}{4 \pi} \frac{\partial}{\partial y}\left[\frac{x}{\rho}\left(\lambda z \frac{e^{i k_{v} s}}{s}-z \frac{e^{i k_{h} r}}{r}\right)\right] \\
= & \frac{i \omega \mu M}{4 \pi} \frac{x y z}{\rho^{2}}\left[-\frac{2}{\rho^{2}}\left(\lambda \frac{e^{i k_{y} s}}{s}-\frac{e^{i k_{h} r}}{r}\right)+\frac{\lambda e^{i k_{v} s}}{s^{3}}\left(i k_{v} s-1\right)-\frac{e^{i k_{h} r}}{r^{3}}\left(i k_{h} r-1\right)\right],(4) \\
E_{y}= & \frac{i \omega \mu M}{4 \pi}\left\{\frac{\partial}{\partial z}\left(\frac{1}{\lambda} \frac{e^{i k_{v} s}}{s}\right)-\frac{\partial}{\partial x}\left[\frac{x}{\rho^{2}}\left(\lambda z \frac{e^{i k_{v} s}}{s}-z \frac{e^{i k_{h} r}}{r}\right)\right]\right\} \\
= & \frac{i \omega \mu M}{4 \pi}\left\{\frac{\lambda z e^{i k_{v} s}}{s^{3}}\left(i k_{v} s-1\right)-\frac{\left(y^{2}-x^{2}\right) z}{\rho^{4}}\left(\lambda \frac{e^{i k_{v} s}}{s}-\frac{e^{i k_{h} r}}{r}\right)-\right. \\
= & -\frac{i \omega \mu M \lambda}{4 \pi} \frac{y e^{i k_{v} s}}{s^{3}}\left(i k_{v} s-1\right) \cdot(6) \\
& \left.\frac{x^{2} z}{\rho{ }^{2}}\left[\frac{\lambda e^{i k_{v} s}}{s^{3}}\left(i k_{v} s-1\right)-\frac{e^{i k_{h} r}}{r^{3}}\left(i k_{h} r-1\right)\right]\right\}, \\
E_{z}= & -\frac{i \omega \mu M \lambda}{4 \pi} \frac{\partial}{\partial y}\left(\frac{e^{i k_{v} s}}{s}\right)
\end{aligned}
$$

When $\lambda=1$ (i.e., isotropic case), equations (4) - (6) reduce to

$$
\begin{aligned}
& E_{x}=0, \\
& E_{y}=\frac{i \omega \mu M}{4 \pi} \frac{z e^{i k r}}{r^{3}}(i k r-1), \\
& E_{z}=-\frac{i \omega \mu M}{4 \pi} \frac{y e^{i k r}}{r^{3}}(i k r-1) .
\end{aligned}
$$


Equation (4) shows that there exists an electric component $\left(E_{x}\right)$ parallel to magnetic dipole in anisotropic media while $E_{x}=0$ in an isotropic whole space. Equation (6) shows that the vertical component of the electric field disappears as $\lambda \rightarrow \infty$. This indicates that the electric field tends to take a horizontal path as the coefficient of anisotropy increases. It is the generation of this $E_{x}$ component, coupled with the fact that the electric fields are almost entirely horizontal for very large coefficients of anisotropy that leads to the rather complicated electric field and current flow-paths that are demonstrated below.

Once the electric fields are obtained, electric currents can be easily computed as

$$
\begin{aligned}
& j_{x}=\sigma_{h} E_{x}, \\
& j_{y}=\sigma_{h} E_{y}, \\
& j_{z}=\sigma_{v} E_{z} .
\end{aligned}
$$

Because in most transverse isotropic formations $\sigma_{h}>\sigma_{v}$, the ratio of the vertical to horizontal currents will be less than the ratio of the comparable electric fields; this leads to additional interesting effects which will be noted below.

\section{VISUALIZATION OF THE ELECTRIC FIELDS}

To investigate how the electric field varies spatially, and to determine how increasing anisotropy affects the resulting fields, equations 4,5 , and 6 are employed to determine the vector field throughout a 3D volume surrounding a horizontal magnetic dipole source. A transmitter dipole of unit moment is located 
at the origin and is directed in $x$-direction. The transmitter is operating at a frequency of $25 \mathrm{kHz}$, and the horizontal conductivity is fixed through all of the following simulations to be $1.0 \mathrm{~S} / \mathrm{m}$.

In induction logging only the conductivity very near to the borehole is resolved, and thus the volume that is investigated in this study is rather small. Here this region is limited, depending on the type of plot, to regions of either $3 \times 3 \mathrm{~m}^{2}$, or $4 \times 4 \times 2 \mathrm{~m}^{3}$. Considering the operating frequency above, this means that the fields are located in the quasi-static field where the imaginary or out-of-phase component is much larger than the in-phase component. Thus in the plots below, only the imaginary component is plotted.

For a more thorough understanding of the fields, results are shown using both $2 \mathrm{D}$ and $3 \mathrm{D}$ visualization. Electric fields in the $y z$-plane at $\mathrm{x}=0 \mathrm{~m}$ and $x y$-plane at $\mathrm{z}=0.1 \mathrm{~m}$ are shown in 2D (for example, Fig. $2 \mathrm{a}$ and $2 \mathrm{~b}$ ). Arrows within these plots indicate the direction of the electric fields at a particular point in that plane, and background color represents the amplitude. Note that the electric fields shown in these $2 \mathrm{D}$ plots do not include any information about the third component, which is perpendicular to the plane.

The 3D graphics, for example Fig.2 $c$, shows streamlines of electric fields emanating from five points $(0.0,0.0,0.4),(0.1,0.0,0.4),(0.2,0.0,0.4),(0.3,0.0,0.4)$ and $(0.4,0.0,0.4)$. The tangent at one point on a streamline is parallel to the electric field at that particular point. The isosurface describing a total electric field amplitude of $0.01 \mathrm{~V} / \mathrm{m}$ is also included. The arrow at the origin in these $3 \mathrm{D}$ renderings represents the horizontal magnetic dipole. The horizontal plane of $z=0$ is also shown in these 3D plots.

As expected, for the isotropic case (Fig. 2) the electric fields form a circular pattern about the source. This is especially evident in the 2D yz-cross section plot (Fig. 2a) which includes the source location. Note that those induced circular electric fields are opposite to the exciting currents in the transmitting coil. In the $x y$-plane above the source the circular pattern translates to arrows that are all in the same direction (Fig. 2b). The amplitudes of electric fields in both planes show the same circular pattern, but the electric fields in the $x y$-plane decrease faster away from the origin than the fields in the $y z$-plane as the distance from the source increases.

The circular nature of the fields is also apparent in the 3D plot given in Figure $2 \mathrm{c}$. Note that the streamlines form coaxially aligned circles about the source, and the constant amplitude isosurface takes 
on a donut shape. This simple case provides a reference against which one can determine the effects of increasing the formation anisotropy as discussed below.

For moderately anisotropic media where $\lambda^{2}=2$ and 5 (Fig. $3 \& 4$ ), the pattern of electric fields in the $y z$ plane becomes elliptical with elongation in $y$-direction. The electric fields tend to take more of a horizontal path as $\lambda^{2}$ increases (Fig. 3a \& Fig. 4a). However, an $x$-component of the field also develops immediately and becomes larger as the coefficient of anisotropy increases (Fig. 3b \& Fig. 4b). Thus the electric fields in the $x y$-plane are no longer simply parallel to $y$-direction. Rather, the fields converge about the origin and then diverge away from this location. For $\lambda^{2}=5$, the electric fields in the $x y$-plane change direction along $y=O$ (Fig. 4b). For the three-dimensional plots, the electric field streamlines are elongated in both directions of the $y$-axis, and away from the $y z$-plane (Fig. $3 c \& 4 c$ ). This causes the isosurface of the total electric field to be extended horizontally.

As the coefficient of anisotropy increases $\left(\lambda^{2}=10,20\right.$, and 100), the electric fields in the $y z$-plane become almost entirely horizontal (Fig. 5a, 6a and 7a), when $\lambda^{2}=100$ (Fig. 7a) the only vertical fields that occur are immediately adjacent to the plane of $z=0$. The electric fields in the $x y$-plane have a pattern in which electric fields emit from the origin, spread out, and converge back to the origin (Fig. $5 b$, $6 \mathrm{~b}$, and $7 \mathrm{~b}$ ). Figures $5 \mathrm{~b}, 6 \mathrm{~b}$, and $7 \mathrm{~b}$ clearly indicate that amplitude of horizontal electric fields becomes larger as anisotropy increases.

Figures $5 \mathrm{c}, 6 \mathrm{c}$, and $7 \mathrm{c}$ show electric field streamlines for $\lambda^{2}=10,20$ and 100 . Note that the electric field streamlines begin 'close' within a horizontal plane, do not cross through the $x y$-plane of $z=0$ for $\lambda^{2}$ larger than 20 (Fig. $6 \mathrm{c}$ and $7 \mathrm{c}$ ). The isosurface of the total electric field extends increasingly in the horizontal direction, especially in the $y$-direction, and also shows that the induced electric fields are restrained in an elliptic-cylinder-like region elongated in $y$-direction.

In summary, as the coefficient of anisotropy increases, the horizontal electric field becomes larger while the vertical component of the field decreases. In media with a large coefficient of anisotropy, almost all of the electric field is horizontal except regions close to the $x y$-plane of $z=0$. In addition, there exists an $x$-component of the electric field in anisotropic media, which is zero in an isotropic whole space. 


\section{VISUALIZATION OF THE CURRENTS}

As mentioned in Section 2, the current density will produce a different pattern when compared to the electric field due to the fact that the currents are the fields premultiplied by the conductivities as outlined in equations (10) - (12). To illustrate the differences that result, the currents have been plotted in the same form as employed in Section 3. Since the horizontal conductivity is fixed in this study and equals to $1.0 \mathrm{~S} / \mathrm{m}$, plots of electric currents in the $x y$-plane exhibit the same patterns as those of electric fields. In addition, the figures for isotropic case $\left(\lambda^{2}=1\right)$ are the same as shown in Figure 3. Thus here only the electric currents in the $y z$-plane and 3D electric current streamlines for $\lambda^{2}=2,5,10,20$ and 100 are presented. A current streamline is the path on which a charge moves. The isosurface in the 3D plot encloses all values greater than $0.01 \mathrm{~A} / \mathrm{m}^{2}$.

In moderately anisotropic media $\left(\lambda^{2}=2 \& 5\right)$, the currents in the $y z$-plane are elliptical and circulate around the source (Fig. 8a and 9a). However, a comparison with Figures 3a and 4a shows that the preference to horizontal of the current flow is greater than that of the fields. For example, for $\lambda^{2}$ values as low as 5 , almost all the current flows horizontally except in regions that are close to, and within the horizontal plane containing the source (Fig. 9a). In 3D plots this is reflected in current streamlines that extend further horizontally away from the origin than electric field streamlines (Fig. 8b and 9b). The most noticeable difference between the isosurfaces of electric fields and currents is that the isosurface of the total electric current is "pinched in" towards the origin along the $x y$-plane, while the isosurface describing the total electric field is convex.

For greater coefficients of anisotropy $\left(\lambda^{2}=10,20\right.$ and 100), the currents flow in the $y z$-plane is almost entirely horizontal (Fig. 10a, 11a, and 12a). The current streamlines continue to extend horizontally and for $\lambda^{2}=10$ begin to form closed paths in a given horizontal plane (Fig. 10b). In fact, as $\lambda^{2}$ increases further beyond 10 to 20 and 100 , the vertical resistivity is so great that all of the current streamlines are closed horizontally (Fig. $11 \mathrm{~b}$ and $12 \mathrm{~b}$ ). The isosurface of the total currents again pinches in the $x y$-plane of $z=0$, but does not change much as $\lambda^{2}$ increases.

\section{DISCUSSION AND CONCLUSIONS}


In this paper the effects of anisotropy on the electric fields and current density generated by a horizontal magnetic dipole have been demonstrated. The electric fields and current paths have been shown to be highly distorted compared to those generated in an isotropic media even for small coefficients of - anisotropy. These distortions can be broken down into three distinct phenomena that appear in transverse isotropic media. The first is that the electric fields and currents take an increasingly horizontal path as the coefficient of anisotropy increases. This is physically intuitive since it is more conductive in the horizontal direction than in the vertical. The second, less intuitive phenomenon that is observed is that the electric fields and currents develop components that are parallel to the moment of the magnetic dipole source, and that this component becomes larger as the coefficient of anisotropy increases. The third phenomenon is that for large coefficients of anisotropy the electric fields and currents form closed loops in horizontal planes rather than in vertical plane for an isotropic whole space. The cause of these two phenomena is not obvious, but it can be understood with the help of a second model.

Consider a horizontal magnetic dipole at the interface of two homogeneous half-spaces, and more specifically, at the surface of the earth. In this case the conductivity of the upper half-space is zero, while it is finite in the earth. Currents will be induced in the earth, but because the boundary conditions at the interface dictate continuity of normal current, vertical currents can not exist that flow from the earth into the air, and thus the vertical field is essentially zero in the earth. Therefore all of the induced currents within the earth must take a horizontal path. The requirement that current is conserved further dictates that a component parallel to the dipole moment develops in order to form a system of closed current loops. The final step in this simple analysis is to consider an anisotropic media as stratified formation consisting of very thin layers that alternate between conductive and resistive. Kennedy (1999) shows that such a model will behave like a transverse isotropic model if thickness of each layer is smaller than the tool resolution.

In the end the analysis presented may not help much in the direct interpretation of induction logging data. However, given the fact that the scattered magnetic fields measured by induction tools are generated by the current patterns presented here, the analysis should be helpful to physically understand electromagnetic induction in anisotropic media. 


\section{Acknowledgments}

This work was performed at Sandia National Laboratories and the University of Wisconsin-Madison with funding provided by the Department of Energy's National Gas and Oil Technology Partnership. Sandia is a multi-program laboratory operated by Sandia Corporation, a Lockheed Martin Company, for the United States Department of Energy under Contract DE-AC04-94AL85000. Industrial sponsors of this project include Mobil, Chevron, Schlumberger-Doll, Baker-Atlas, Halliburton Oilfield Services, and ElectroMagnetic Instruments, Inc.

\section{References}

Anderson, B.I., Barder, T.D., and Gianzero, S.C., 1998, The effect of crossbedding anisotropy on induction tool response: $39^{\text {th }}$ SPWLA Logging Symposium, Paper B.

Kennedy, W.D., 1999, Resistivity and anisotropy, The Log Analyst, Vol. 40, No. 6, 451-458.

Klein, J.D., Martin, P.R., and Allen, D.F., 1995, The petrophysics of electrically anisotropic reservoirs, SPWLA $36^{\text {th }}$ Annual Logging Symposium, Paper HH.

Kong, J.A., 1972, Electromagnetic fields due to dipole antennas over stratified anisotropic media, Geophysics, 37, 985-996.

Kong, J.A., 1975, Theory of Electromagnetic Waves, New York, Wiley Interscience.

Kunz, K.S., and Moran, J.H., 1958, Some effects of formation anisotropy on resistivity measurements in boreholes: Geophysics, 23, 770-794.

Moran, J.H., and Gianzero, S.C., 1979, Effects of formation anisotropy on resistivity-logging measurements: Geophysics, 44, 1266-1286. 
Moran, J.H., and Kunz, K.S., 1962, Basic theory of induction logging and application to study of two-coil sondes: Geophysics, 26, 829-858.

Sinha, A.K., 1968, Electromagnetic fields of an oscillating magnetic dipole over an anisotropic earth, Geophysics, 33, 346-353.

Tang, C.M., 1979, Electromagnetic fields due to dipoles antennas embedded in stratified anisotropic media, IEEE Transactions on Antennas and Propagation, AP-27, 665-670.

\section{Figure Captions}

Figure 1. Idealized 2-coil induction sonde located in a horizontal well. Transmitter (Tx) and receiver $(\mathrm{Rx})$ coils are coaxially aligned.

Figure 2. Electric fields in isotropic media $\left(\lambda^{2}=1\right)$. (a) Electric fields in $y z$-plane of $x=0$. (b) Electric fields in $x y$-plane of $z=0.1 \mathrm{~m}$. (c) Streamlines of electric fields and isosurface of $0.1 \mathrm{v} / \mathrm{m}$.

Figure 3. Electric fields in anisotropic media of $\lambda^{2}=2$. (a) Electric fields in the $y z$-plane. (b) Electric fields in the $x y$-plane. (c) Streamlines of electric fields and isosurface of $0.1 \mathrm{v} / \mathrm{m}$.

Figure 4. Electric fields in anisotropic media of $\lambda^{2}=5$. (a) Electric fields in the $y z$-plane. (b) Electric fields in the $x y$-plane. (c) Streamlines of electric fields and isosurface of $0.1 \mathrm{v} / \mathrm{m}$.

Figure 5. Electric fields in anisotropic media of $\lambda^{2}=10$. (a) Electric fields in the $y z$-plane. (b) Electric fields in the $x y$-plane. (c) Streamlines of electric fields and isosurface of $0.1 \mathrm{v} / \mathrm{m}$.

Figure 6. Electric fields in anisotropic media of $\lambda^{2}=20$. (a) Electric fields in the $y z$-plane. (b) Electric fields in the $x y$-plane. (c) Streamlines of electric fields and isosurface of $0.1 \mathrm{v} / \mathrm{m}$. 
Figure 7. Electric fields in anisotropic media of $\lambda^{2}=100$. (a) Electric fields in the yz-plane. (b) Electric fields in the $x y$-plane. (c) Streamlines of electric fields and isosurface of $0.1 \mathrm{v} / \mathrm{m}$.

Figure 8. Electric currents in anisotropic media of $\lambda^{2}=2$. (a) Electric currents in the $y z$-plane. (b) Streamlines of electric currents and isosurface of $0.1 \mathrm{~A} / \mathrm{m}^{2}$.

Figure 9. Electric currents in anisotropic media of $\lambda^{2}=5$. (a) Electric currents in the $y z$-plane. (b) Streamlines of electric currents and isosurface of $0.1 \mathrm{~A} / \mathrm{m}^{2}$.

Figure 10. Electric currents in anisotropic media of $\lambda^{2}=10$. (a) Electric currents in the $y z$-plane. (b) Streamlines of electric currents and isosurface of $0.1 \mathrm{~A} / \mathrm{m}^{2}$.

Figure 11. Electric currents in anisotropic media of $\lambda^{2}=20$. (a) Electric currents in the $y z$-plane. (b) Streamlines of electric currents and isosurface of $0.1 \mathrm{~A} / \mathrm{m}^{2}$.

Figure 12. Electric currents in anisotropic media of $\lambda^{2}=100$. (a) Electric currents in the $y z$-plane. (b) Streamlines of electric currents and isosurface of $0.1 \mathrm{~A} / \mathrm{m}^{2}$. 


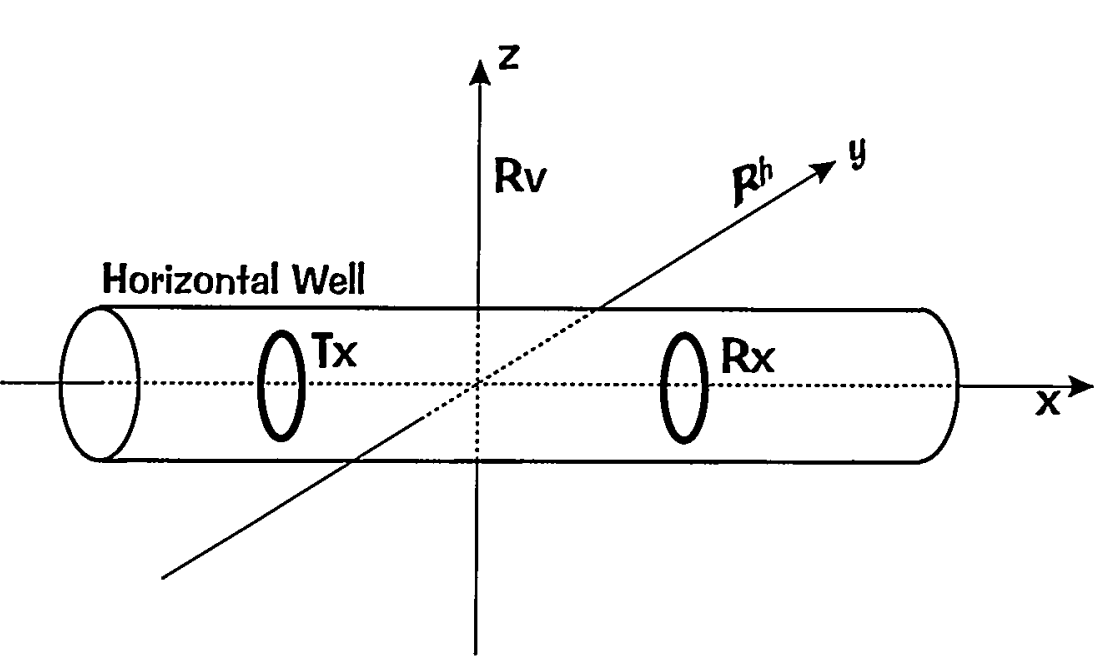



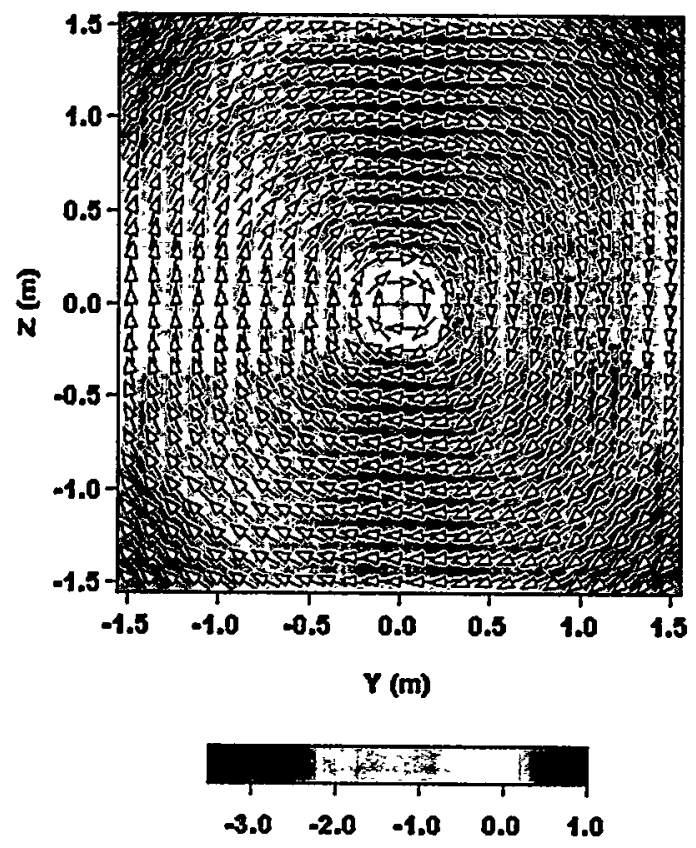

Log Electric Fleld Amplitude
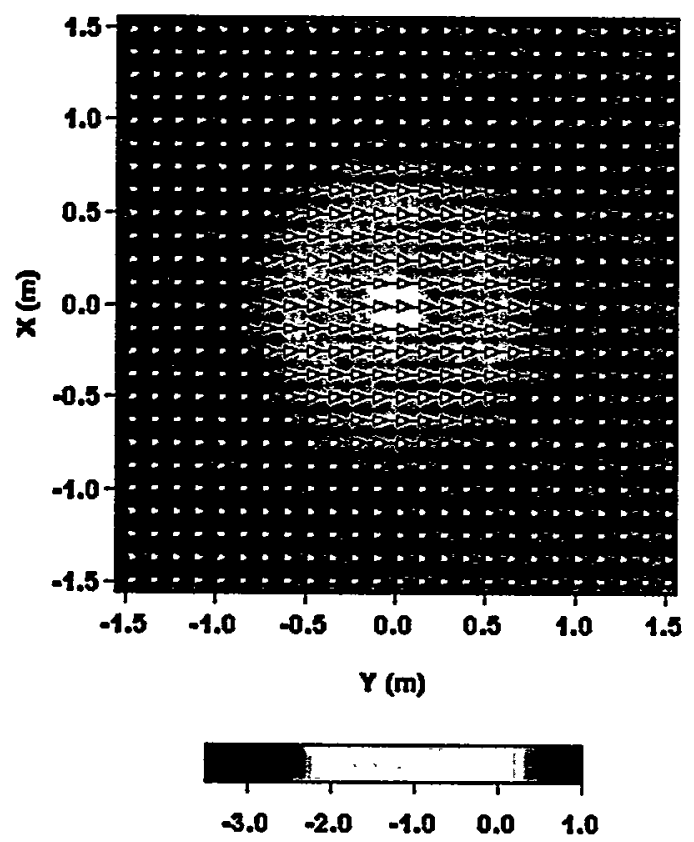

Log Electric Fleld Amplitude

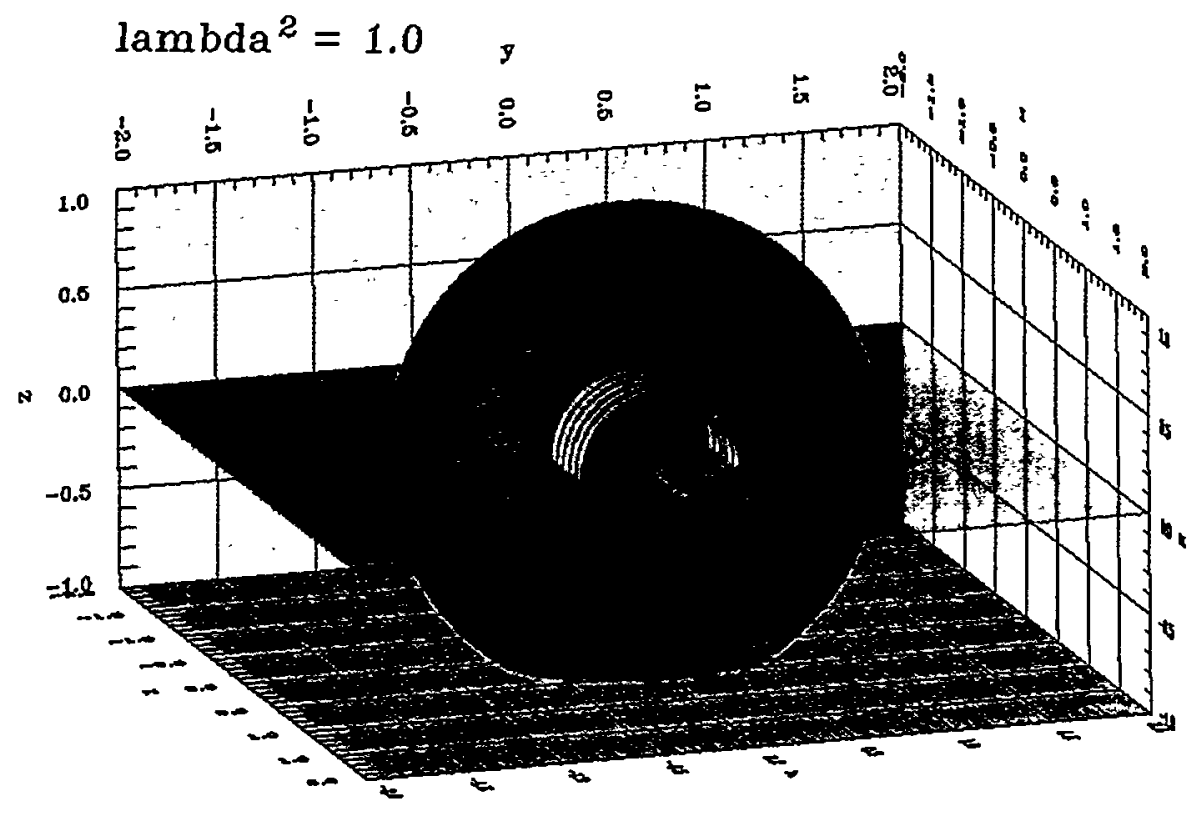

Figure 2 

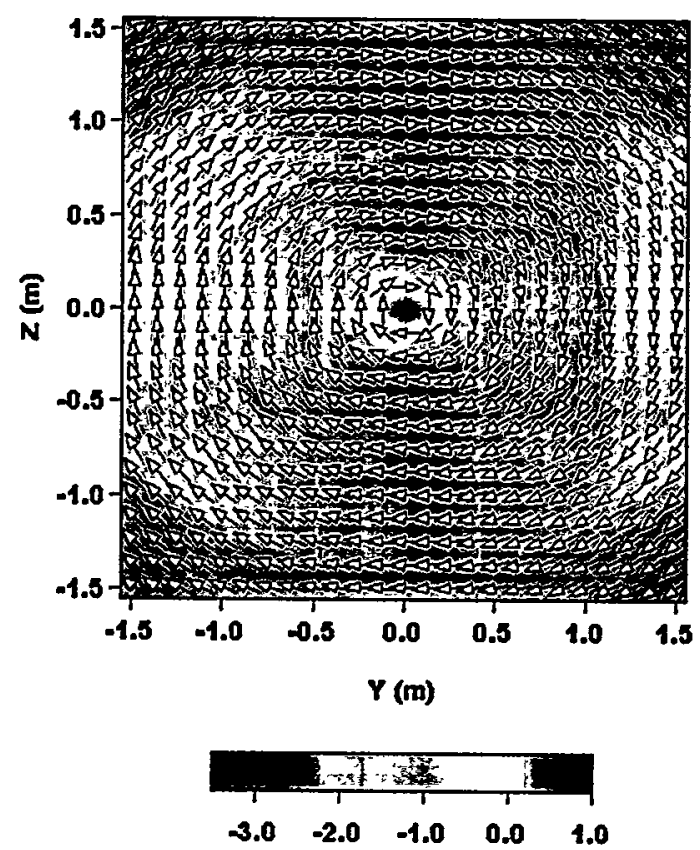

Log Electric Fleld Amplitude
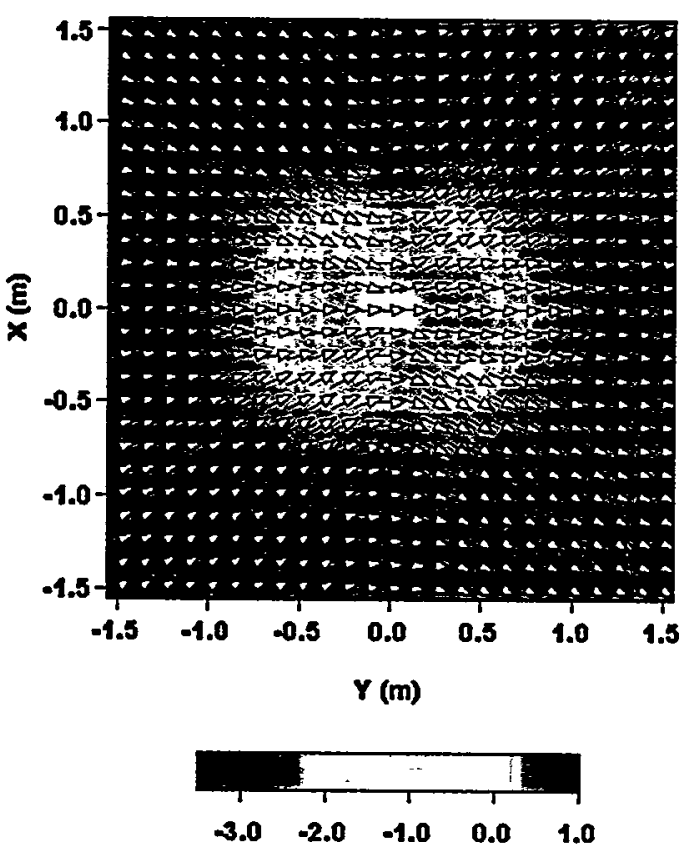

Log Eloctric Field Amplitude

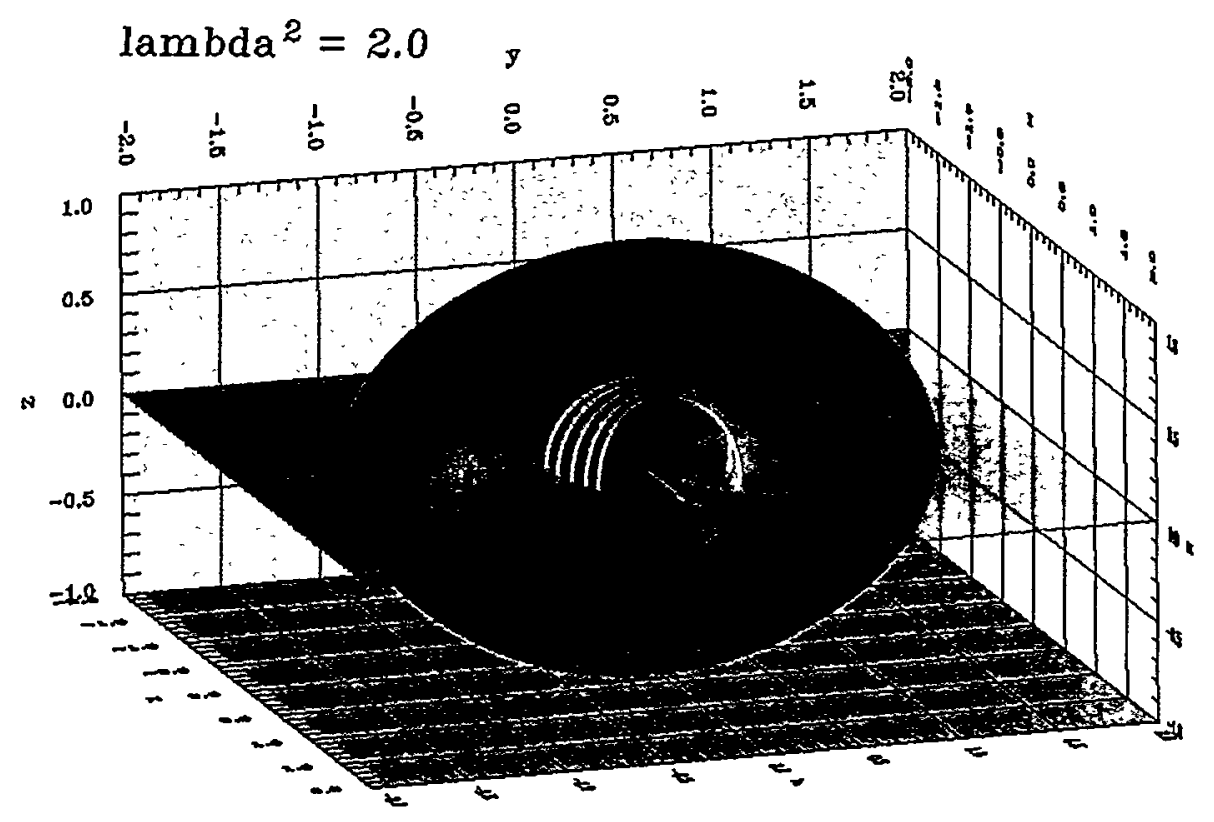

Figure 3 

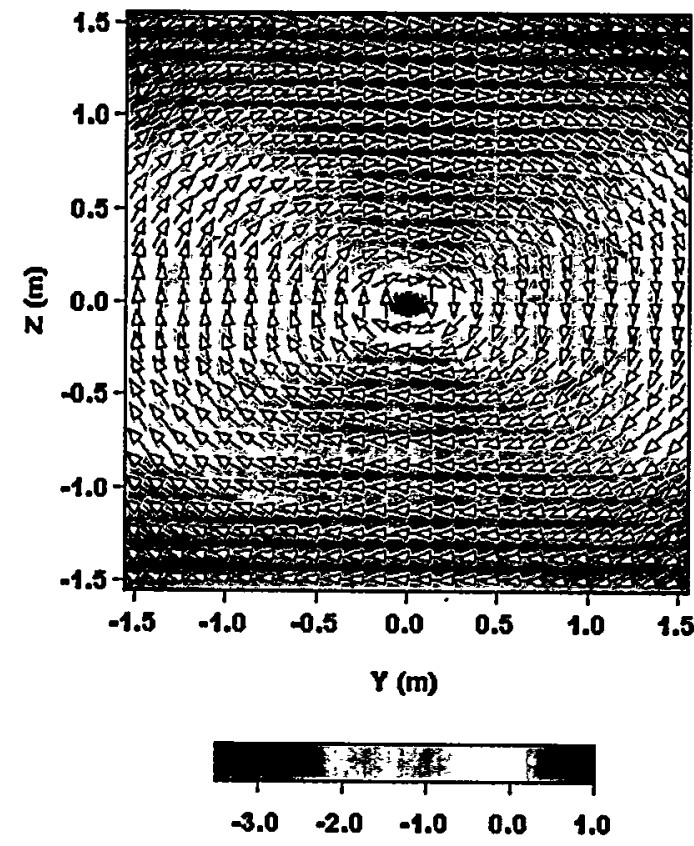

Log Electric Fleld Amplitude
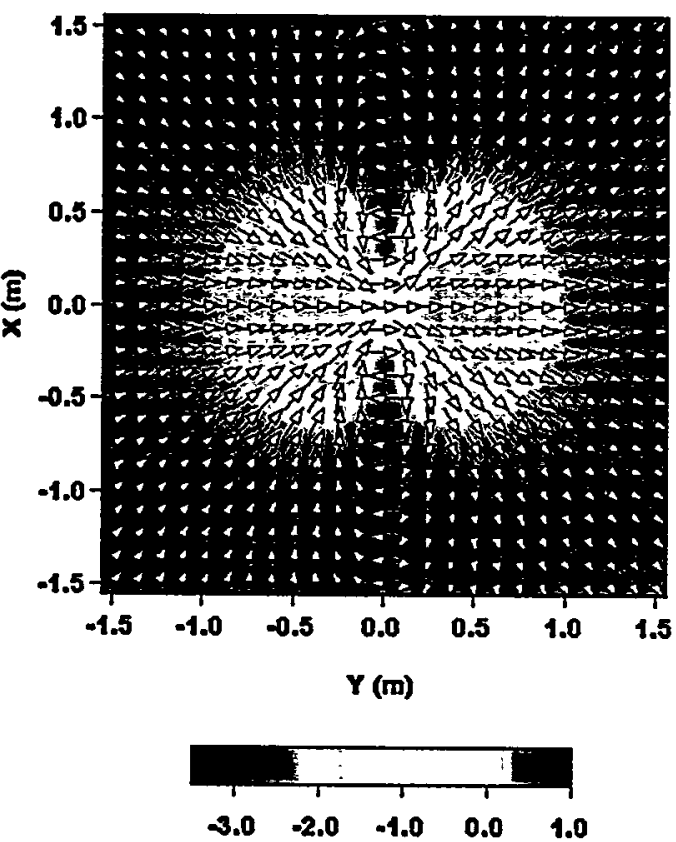

Log Electric Flold Amplitude

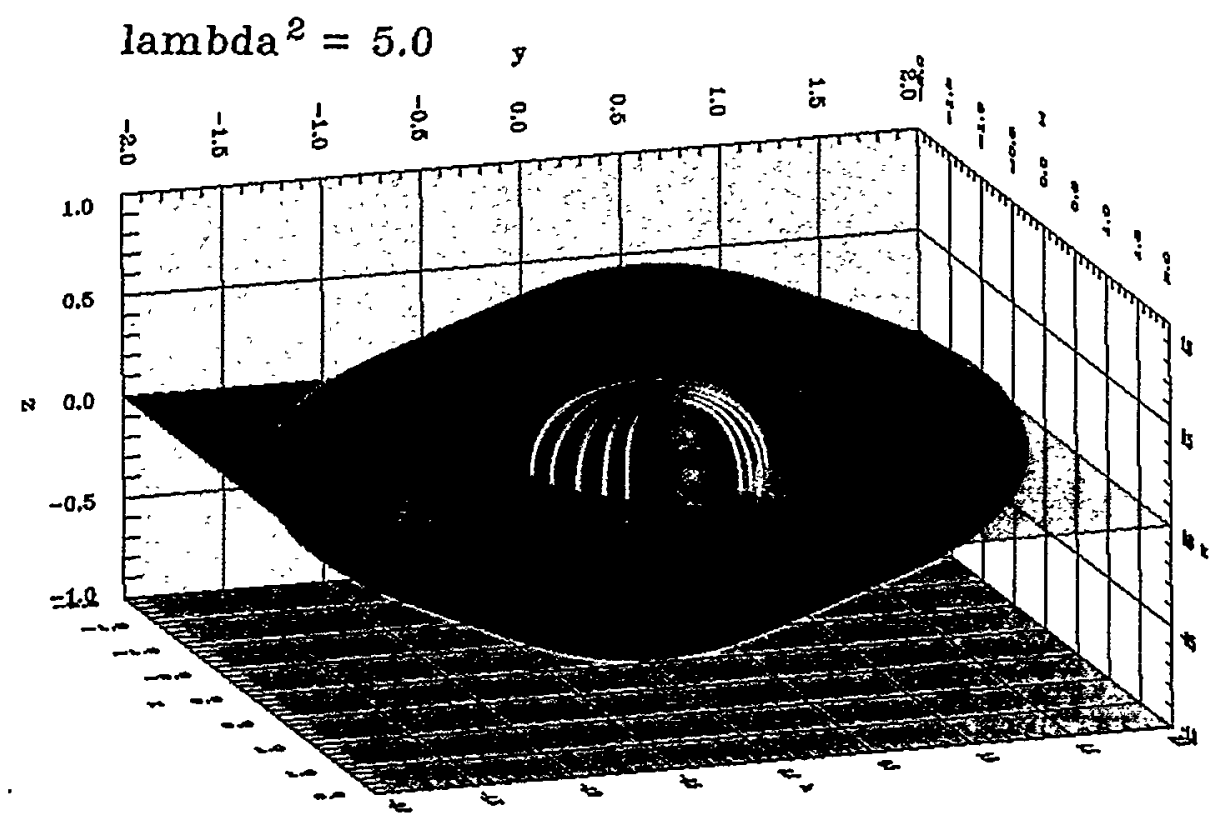

Figure 4 

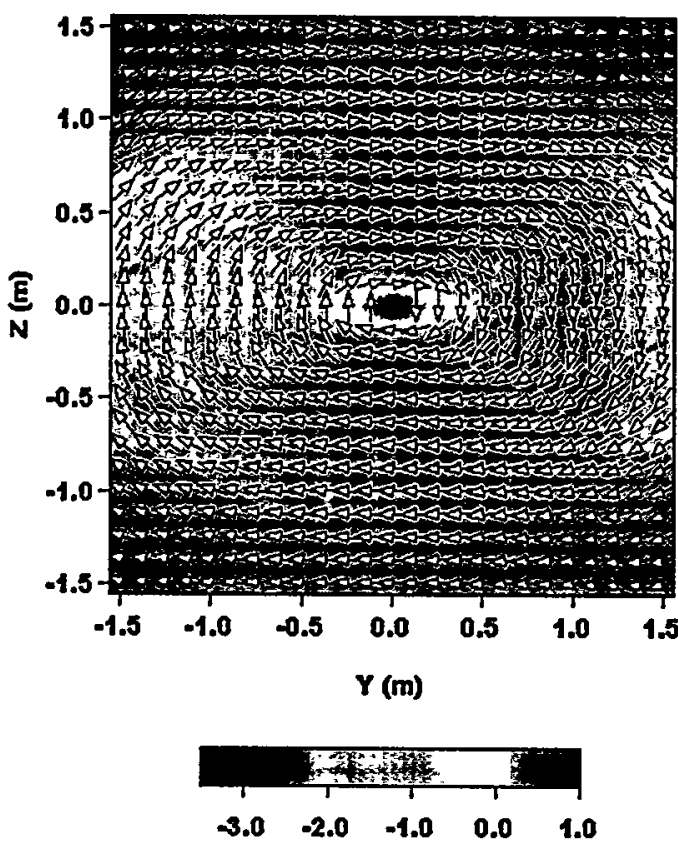

Log Electric Fleld Amplitude
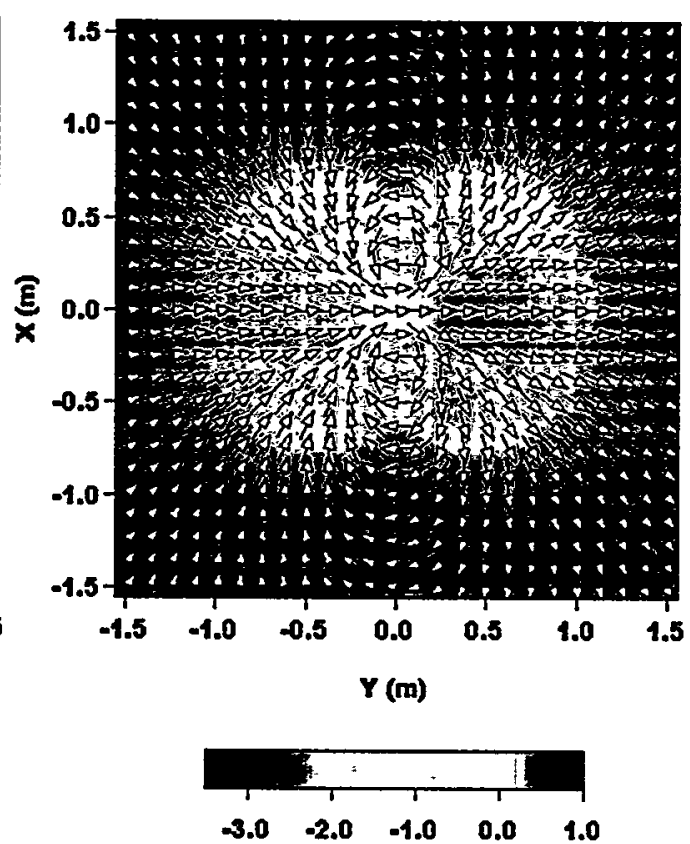

Log Electric Fleld Amplitude

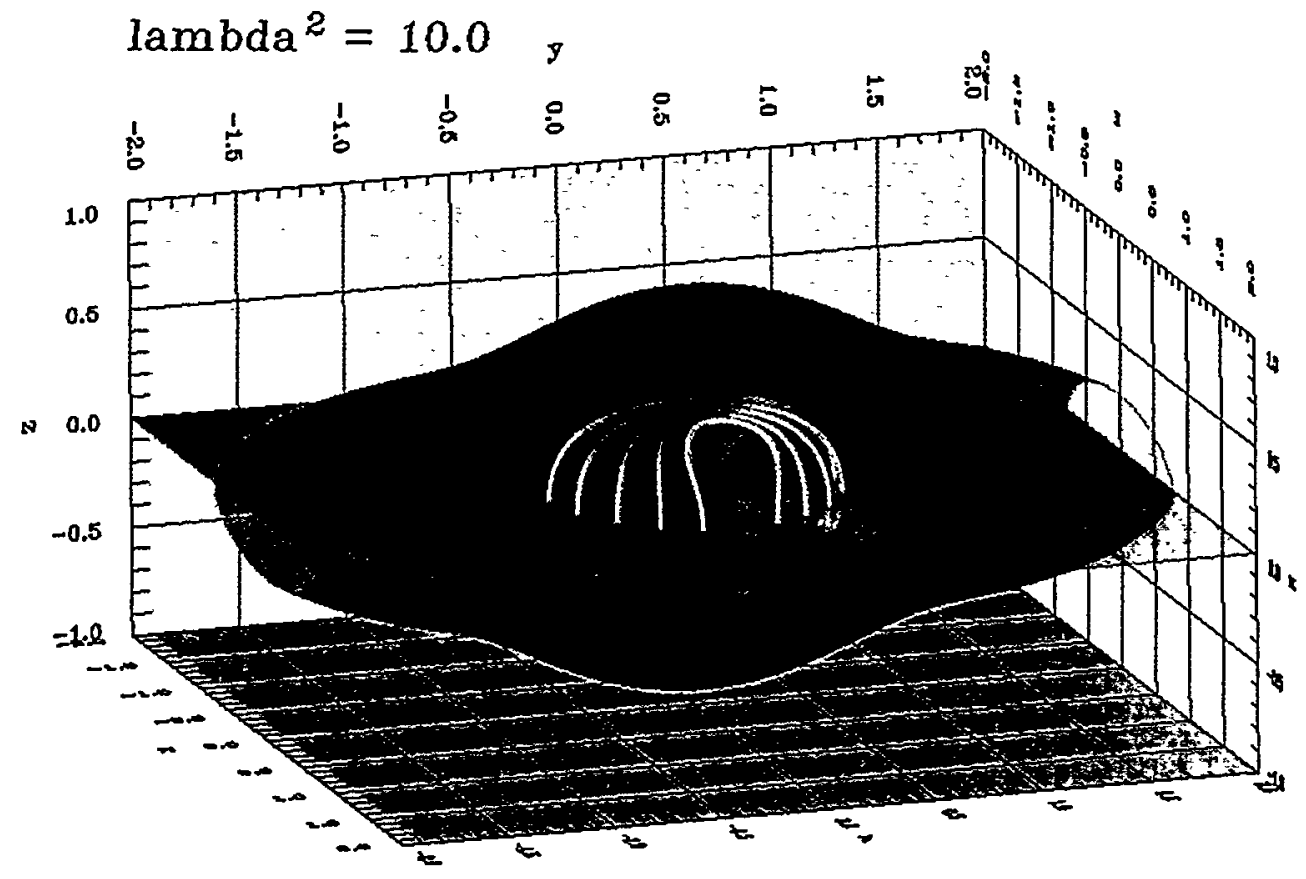

Figure 5 

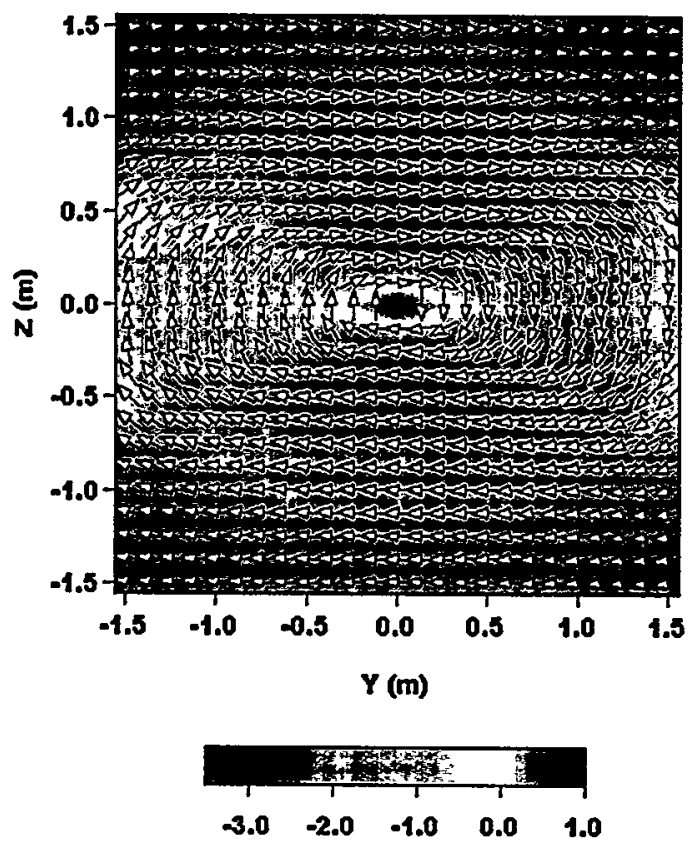

Log Electric Fleld Amplitude
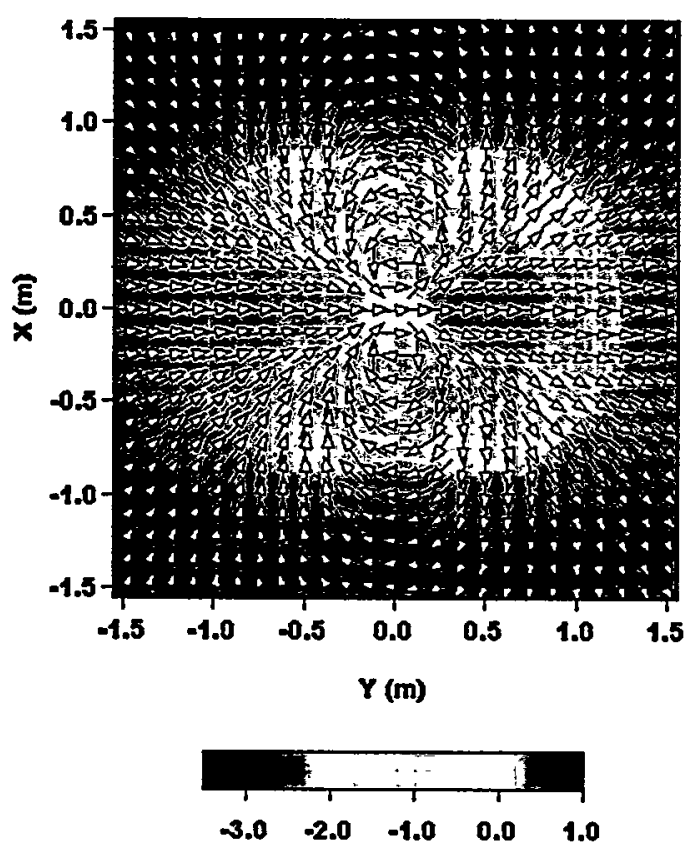

Log Electric Fleld Amplitudo

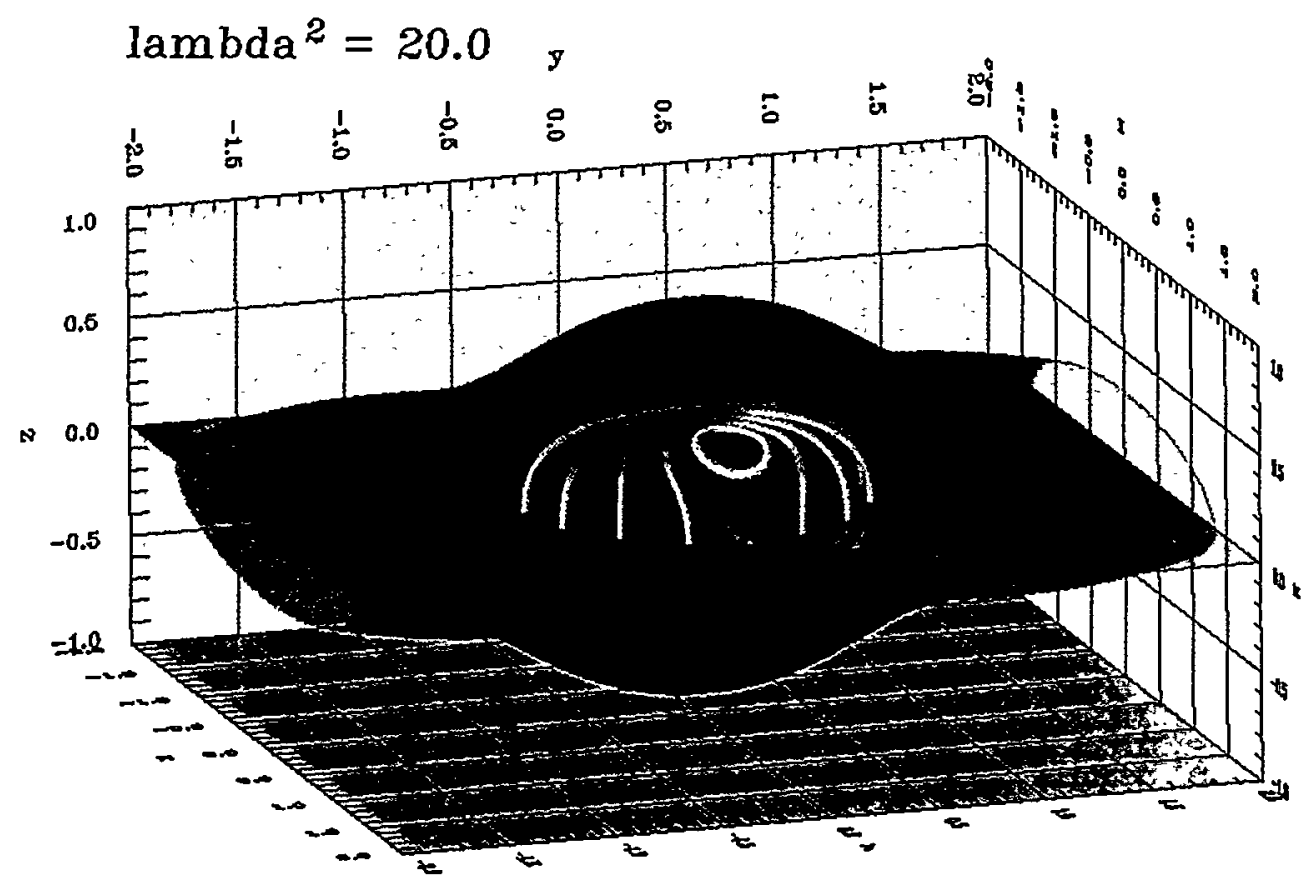

Figure 6 

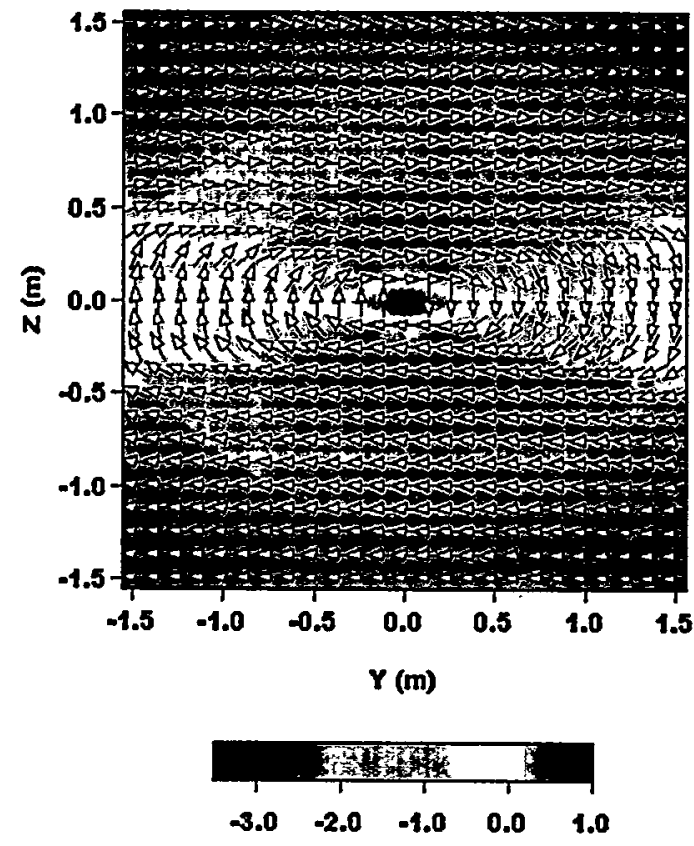

Log Electric Fleld Amplitude
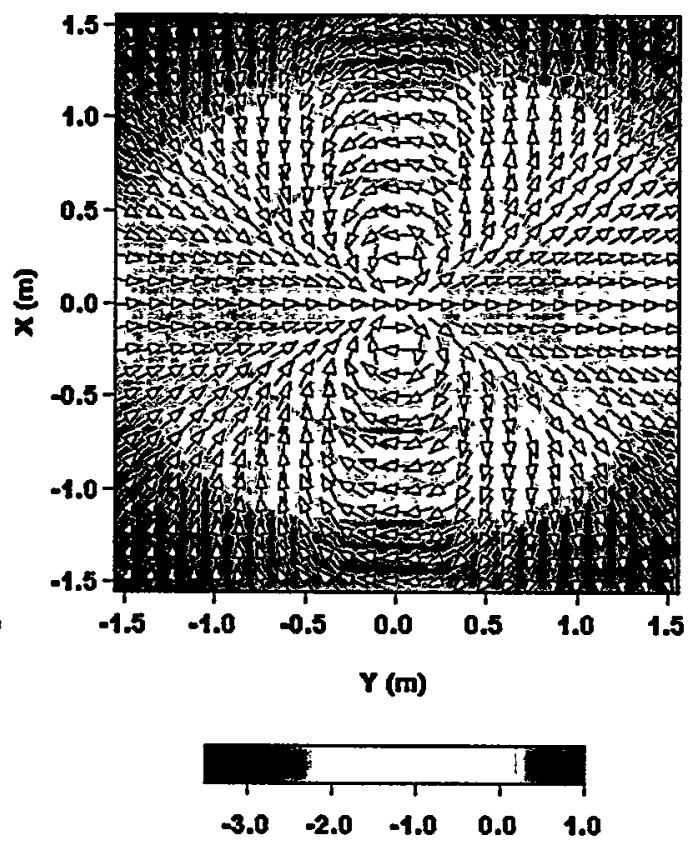

Log Electric Field Amplitude

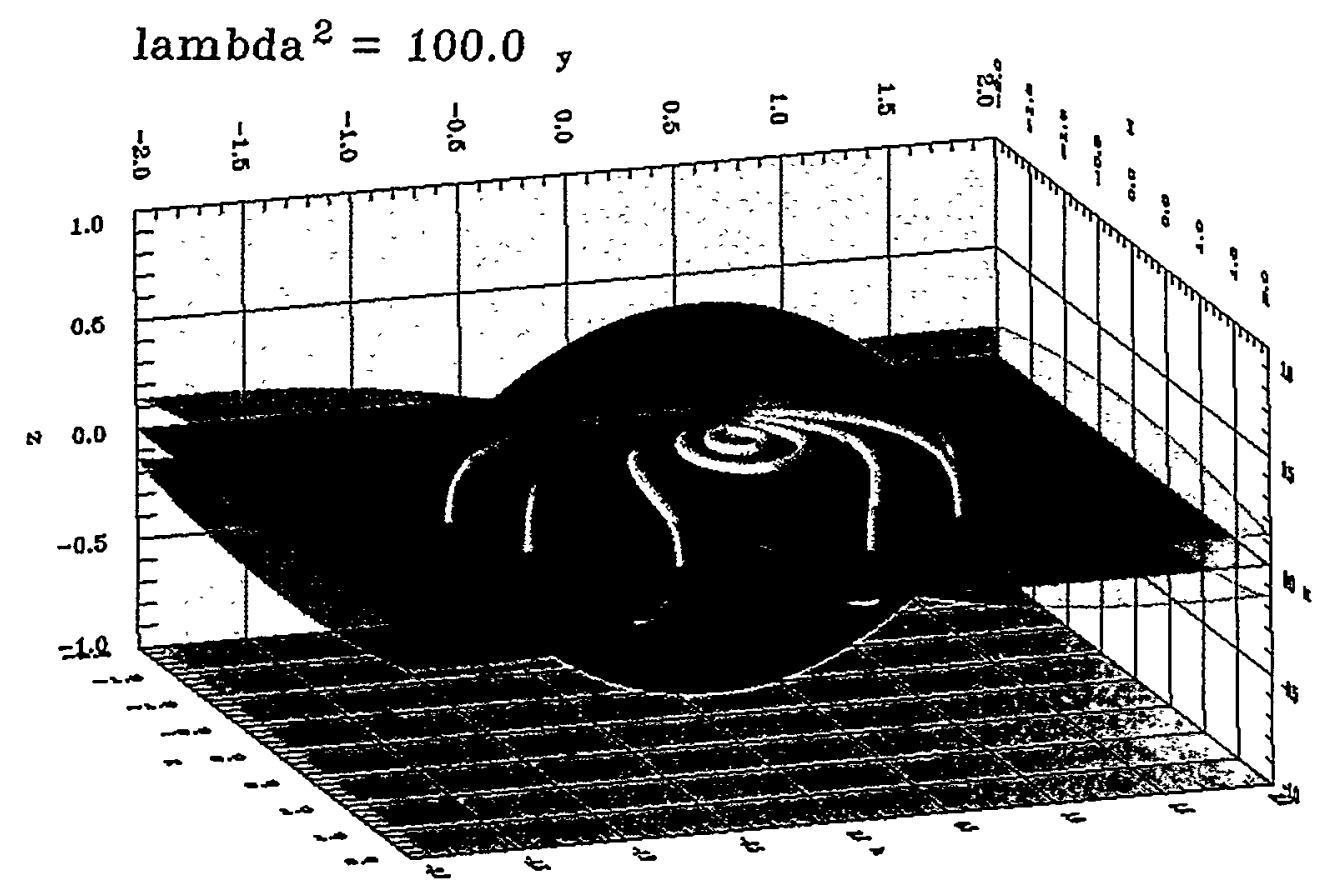

Figure 7 

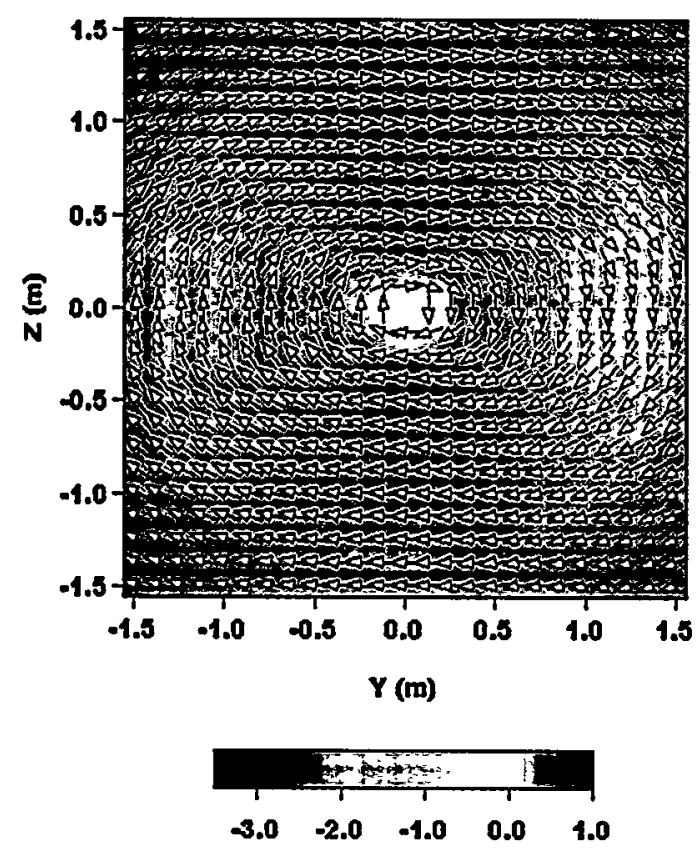

Log Current Density Amplitudo

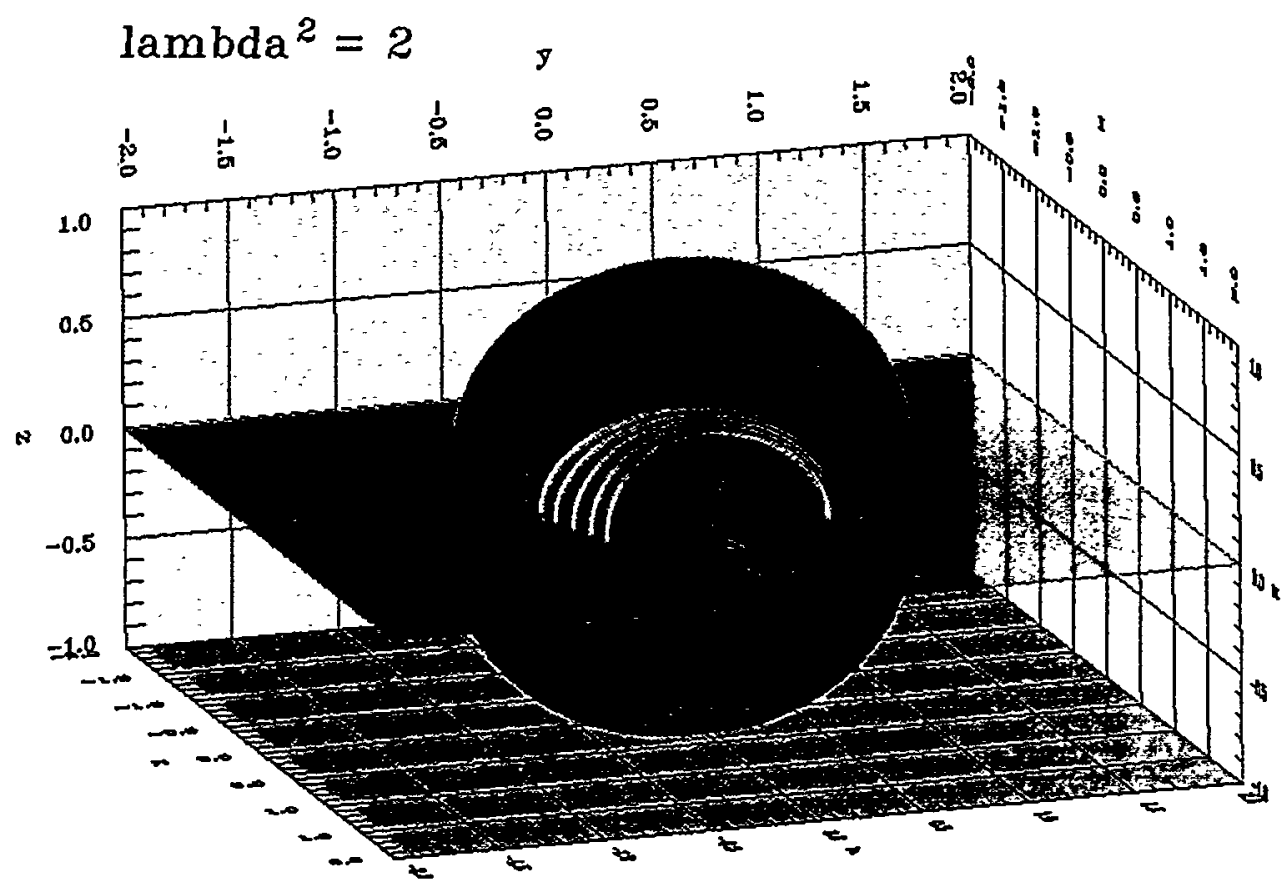

Figure 8 

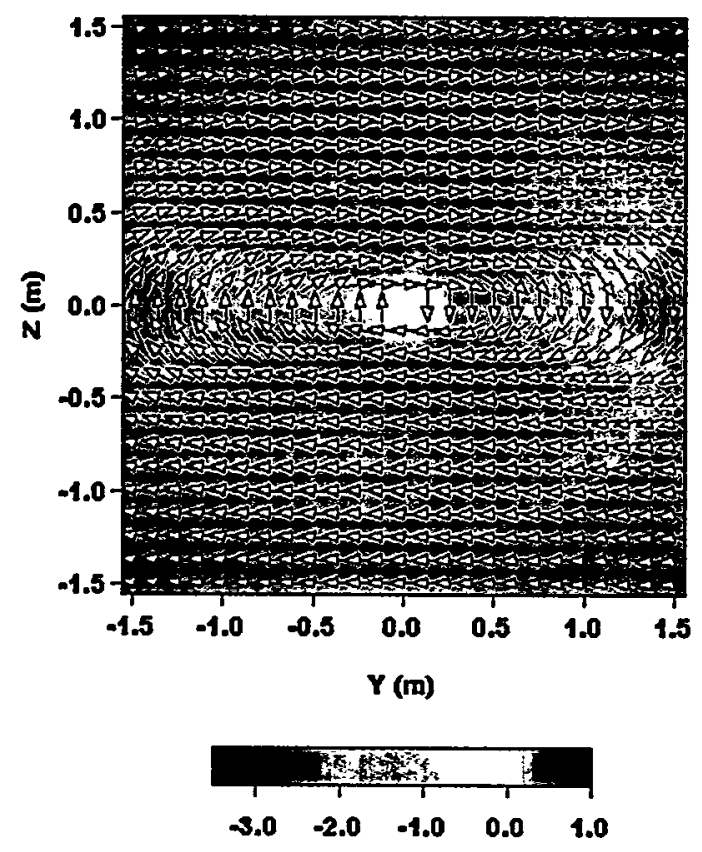

Log Curront Density Amplitudo

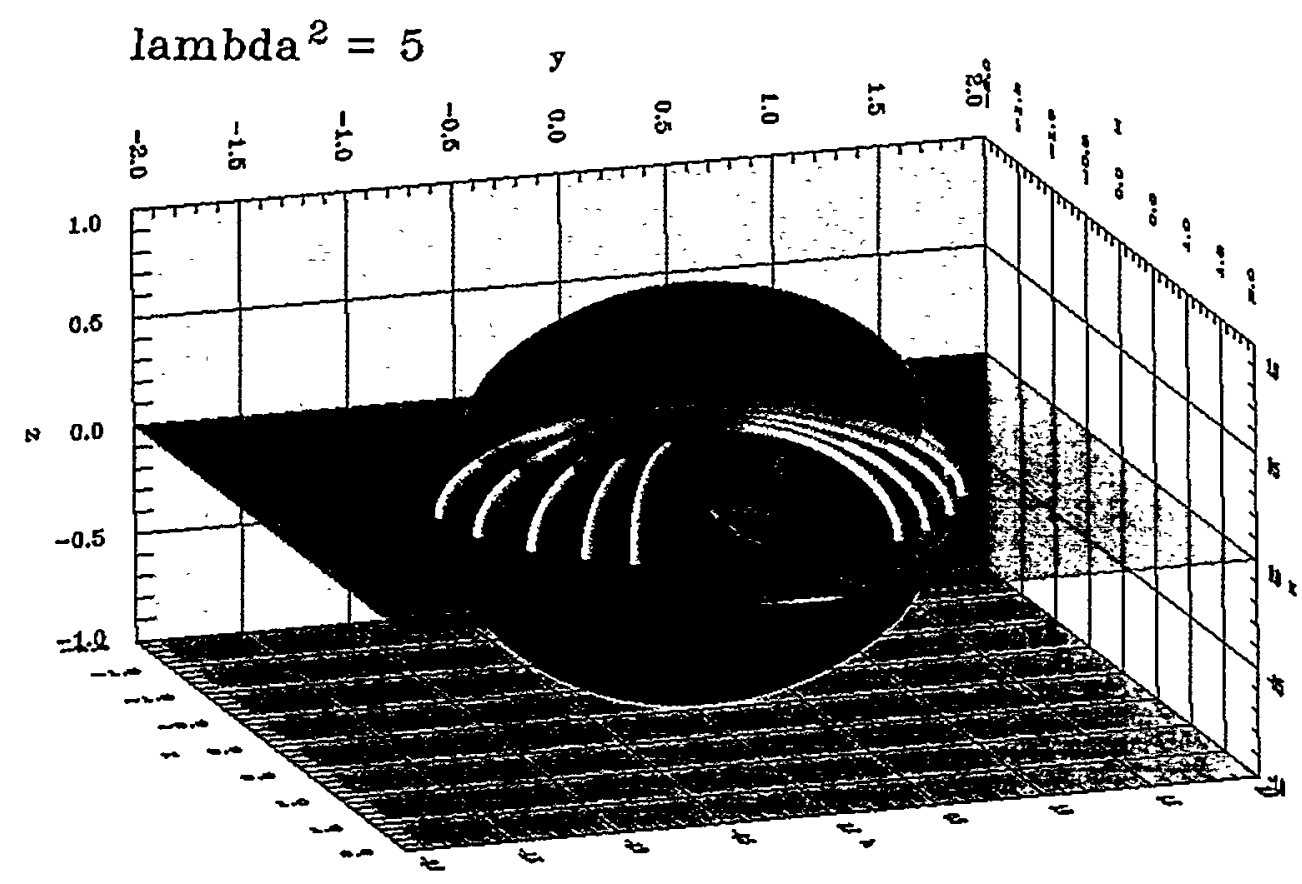

Figure 9 

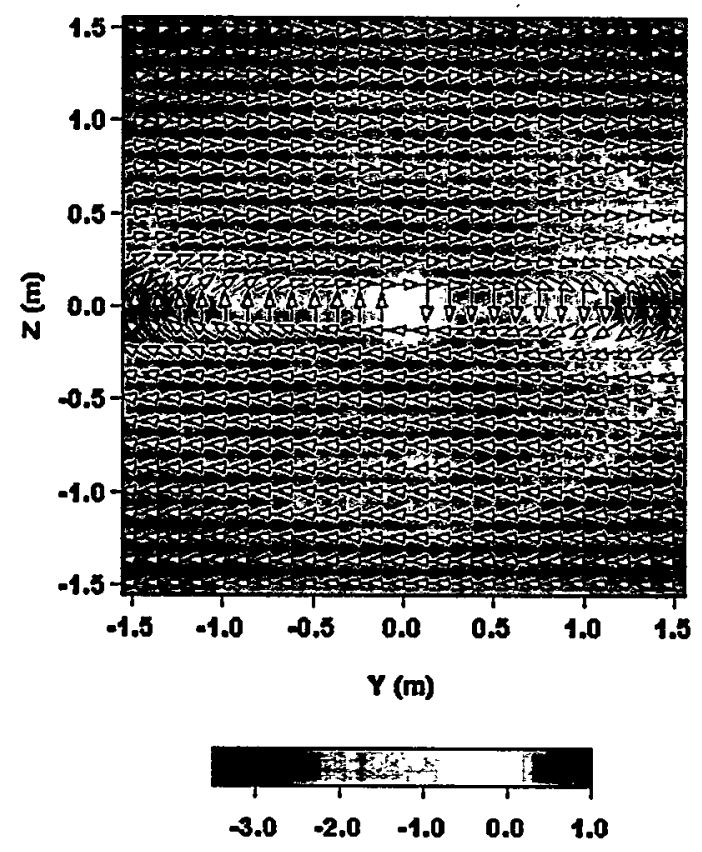

Log Current Density Amplitude

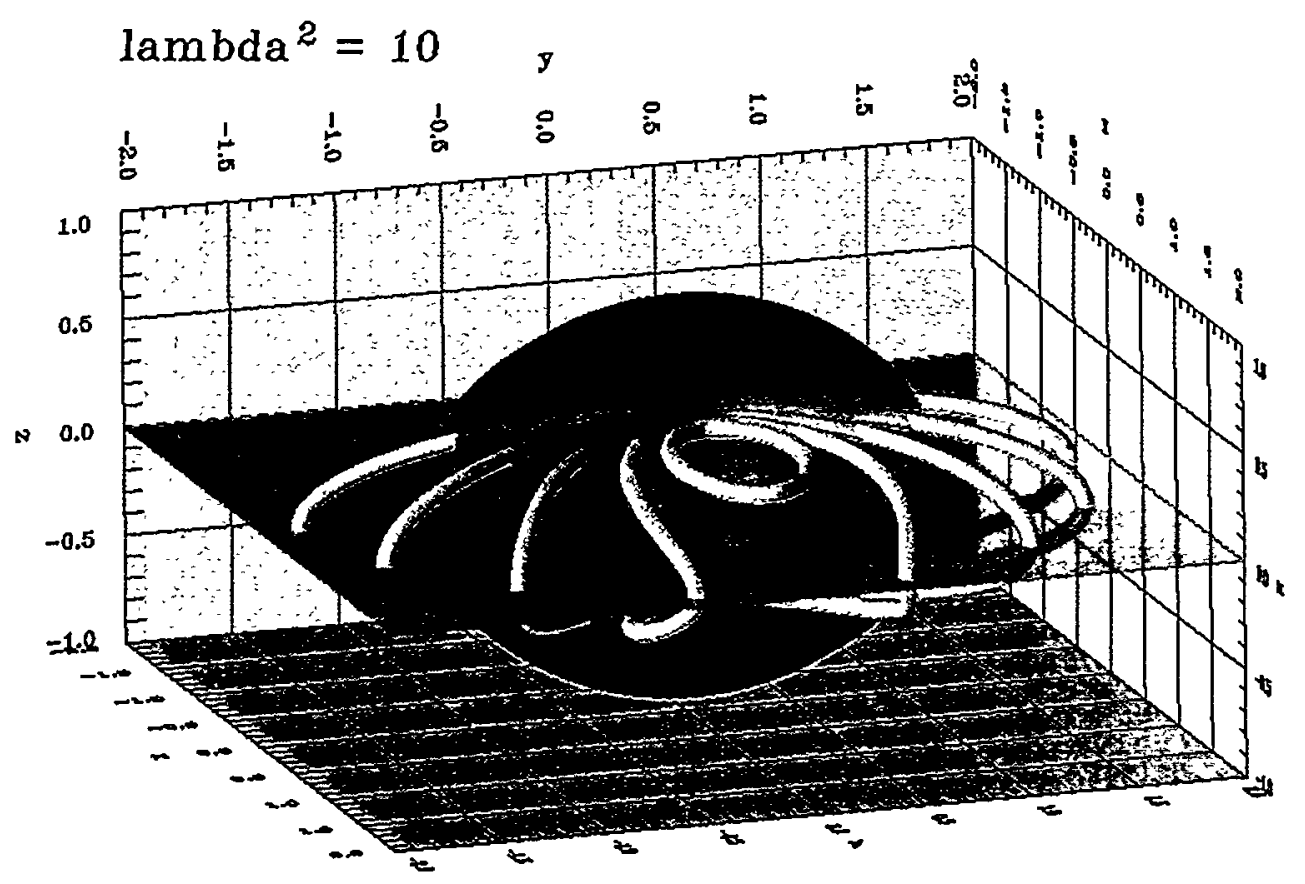

Figure 10 

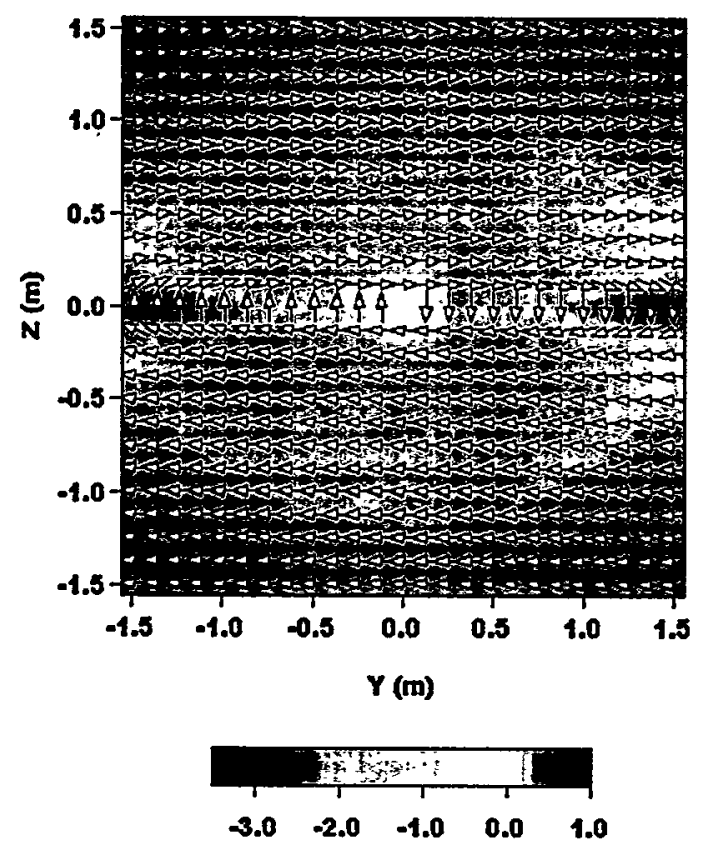

Log Current Density Amplitudo

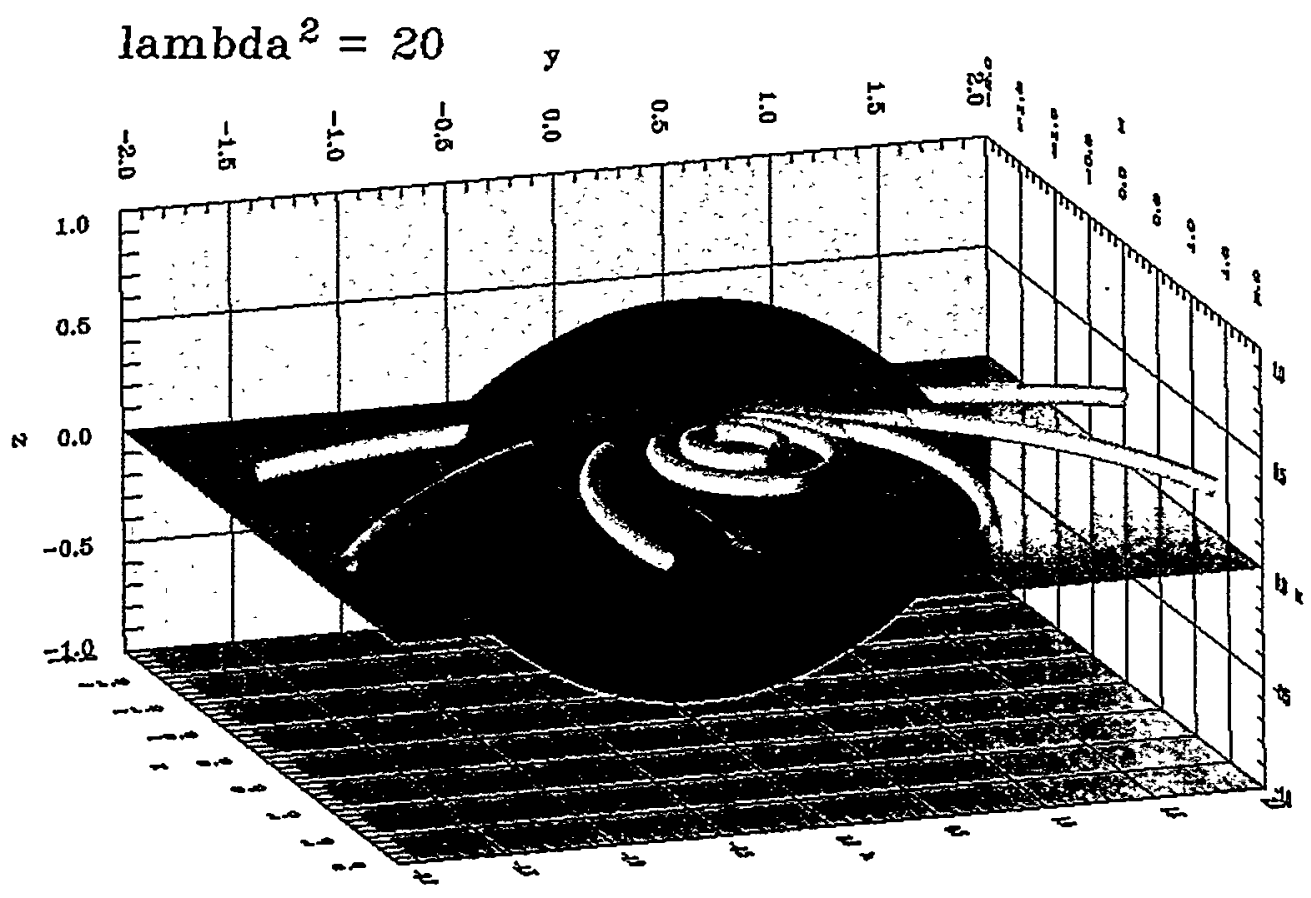

Figure 11 

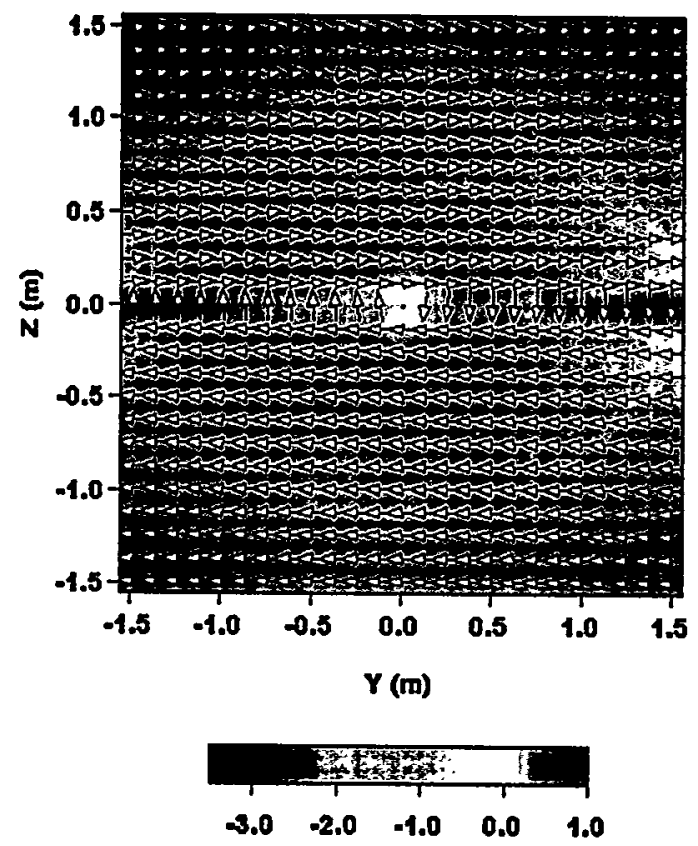

Log Current Density Amplltude

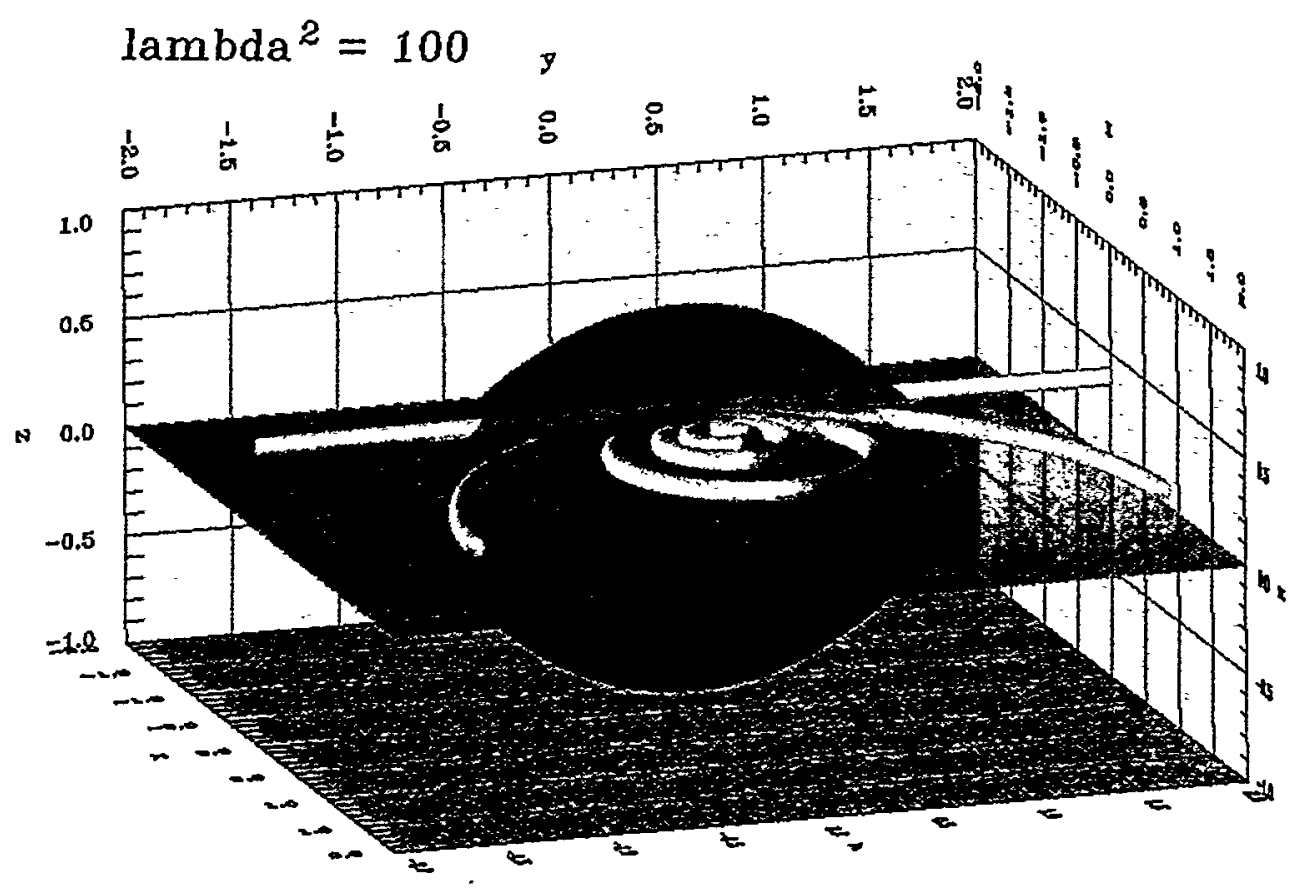

Figure 12 